Фролова Н.Ф. ${ }^{1}$, Ким И.Г.1,2,

Ушакова А.И. ${ }^{1}$, Усатюк C.C. ${ }^{1}$, Артюхина А.Ю. ${ }^{1}$, Исхаков P.T. ${ }^{1}$, Варясин В.В. ${ }^{1}$, Синявкин A.0. ${ }^{1}$, Аьякова Е.Н. ${ }^{1}$, ^осс K.Э. ${ }^{1}$, Червинко В.И. ${ }^{3}$, Володина Е.В. ${ }^{2}$, Томилина Н.А. ${ }^{4}$, Крюков Е.В. ${ }^{5}$, Зубкин М.^. ${ }^{1-3}$
${ }^{1}$ Государственное бюджетное учреждение зАравоохранения города Москвы «Городская клиническая больница № 52 Аепартамента зАравоохранения города Москвы", 123182, г. Москва, Российская Федерация

${ }^{2}$ Федеральное бюАжетное учреждение науки «Московский научноисследовательский институт эпидемиологии и микробиологии им. Г.Н. Габричевского" Федеральной службы по налзору в сфере защиты прав потребителей и благополучия человека, 125212, г. Москва, Российская Федерация

${ }^{3}$ Филиал федерального государственного бюАжетного военного образовательного учреждения высшего образования «Военно-меАицинская академия имени С.М. Кирова" Министерства обороны Российской Федерации, 107392, г. Москва, Российская Федерация

${ }^{4}$ Федеральное государственное бюджетное образовательное учреждение высшего образования «Московский государственный медикостоматологический университет имени А.И. ЕвАокимова" Министерства зАравоохранения Российской Федерации, 127473, г. Москва, Российская Федерация

${ }^{5}$ Федеральное государственное бюджетное учреждение «Главный военный клинический госпиталь им. Н.Н. Бурденко" Министерства обороны Российской Федерации, 105229, г. Москва, Российская Федерация
Цель исследования - анализ клинической картины, особенностей течения, факторов риска и возможностей терапии у больных COVID-19, получавших лечение программным гемодиализом.

Материал и методы. В ретроспективном одноцентровом неконтролируемом исследовании участвовал

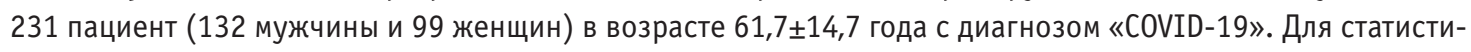
ческой обработки данных использовался пакет программ SPSS (версия 22).

Результаты. Показатель летальности в исследуемой группе составил 31,2\%. Наиболее частой причиной фатального исхода стал острый респираторный дистресс-синдром $(87,5 \%)$. Сравнительный анализ в группах больных с благоприятным и неблагоприятным течением болезни позволил установить, что среди умерших достоверно выше оказались возраст $68,1 \pm 13,2$ года против $58,7 \pm 14,5$ года $(p<0,0001)$ и индекс комор-

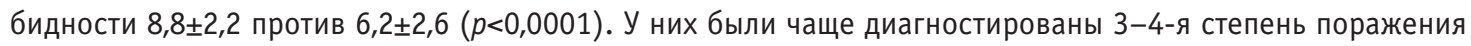
легких, по данным компьютерной томографии (72,2 против 36,5\%, $p<0,0001)$, а минимальный показатель сатурации кислорода составил соответственно $67,6 \pm 12,8$ против $87,8 \pm 10,9 \%$ ( $p<0,0001)$. При использовании многофакторной регрессионной модели Сох независимыми предикторами летального исхода COVID-19 оказались индекс коморбидности и необходимость применения искусственной вентиляции легких. Раннее назначение моноклональных антител к рецептору интерлейкина-6 (в первые 3 сут после госпитализации) у больных с небольшой распространенностью легочного процесса (1-2-я степень поражения легких, по данным компьютерной томографии) оказалось связано с достоверно меньшей частотой фатального течения заболевания.

Заключение. Инфекция SARS-CoV-2 у больных на гемодиализе характеризуется высокой частотой летального исхода. Предикторами тяжелого течения болезни в этой когорте являются высокий индекс коморбидности и применение искусственной вентиляции легких.

Финансирование. Исследование не имело финансовой поддержки.

Конфликт интересов. Авторы заявляют об отсутствии конфликта интересов.

Вклад авторов. Концепция и дизайн исследования - Зубкин М.Л., Фролова Н.Ф., Ким И.Г., Крюков Е.В., Червинко В.И., Томилина Н.А.; сбор и обработка материала - Ушакова А.И., Усатюк С.С., Артюхина Л.Ю. Исхаков Р.Т., Варясин В.В., Синявкин Д.О., Дьякова Е.Н., Лосс К.Э., Володина Е.В.; статистическая обработка данных - Ким И.Г.; написание текста - Зубкин М.Л., Крюков Е.В., Фролова Н.Ф.; редактирование - Зубкин М.Л., Ким И.Г., Крюков Е.В.; утверждение окончательного варианта статьи Зубкин М.Л., Крюков Е.В., Фролова Н.Ф., ответственность за целостность всех частей статьи - Фролова Н.Ф., Зубкин М.Л.
Ключевые слова: гемодиализ, COVID-19, SARS-CoV-2 
Для цитирования: Фролова Н.Ф., Ким И.Г., Ушакова А.И., Усатюк С.С., Артюхина Л.Ю., Исхаков Р.Т., Варясин В.В., Синявкин Д.О., Дьякова Е.Н., Лосс К.Э., Червинко В.И., Володина Е.В., Томилина Н.А., Крюков Е.В., Зубкин М.Л. COVID-19 у больных, получающих лечение программным гемодиализом // Инфекционные болезни: новости, мнения, обучение. 2021. Т. 10, № 1. С. 14-23. D0I: https://doi.org/10.33029/2305-3496-2021-10-1-14-23

Статья поступила в редакцию 19.11.2020. Принята в печать 28.12.2020.

\section{COVID-19 in hemodialysis patients}

Frolova N.F. ${ }^{1}$, Kim I.G. ${ }^{1,2}$,

Ushakova A.I. ${ }^{1}$, Usatiuk S.S. ${ }^{1}$,

Artyukhina L. Yu. ${ }^{1}$, Iskhakov R.T. ${ }^{1}$,

Varyasin V.V. ${ }^{1}$, Sinyavkin D.0. ${ }^{1}$,

Diakova E.N. ${ }^{1}$, Loss K.E. ${ }^{1}$,

Chervinko V.I. ${ }^{3}$, Volodina E.V. ${ }^{2}$,

Tomilina N.A. ${ }^{4}$, Kryukov E.V. ${ }^{5}$,

Zubkin M.L. ${ }^{1-3}$
${ }^{1}$ Moscow City Hospital N 52, 123182, Moscow, Russian Federation

${ }^{2}$ G.N. Gabrichevsky Research Institute for Epidemiology and Micro-

biology, 125212, Moscow, Russian Federation

${ }^{3}$ The Branch of the S.M. Kirov Military Medical Academy, 107392,

Moscow, Russian Federation

${ }^{4}$ A.I. Yevdokimov Moscow State University of Medicine and Den-

tistry of the Ministry of Healthcare of the Russian Federation,

127473, Moscow, Russian Federation

${ }^{5}$ N.N. Burdenko Main Military Clinical Hospital, 105229, Moscow, Russian Federation
Objective. Evaluation of clinical observation, the course, the risk factors, and treatment options for SARS-CoV-2 infection in hemodialysis patients with end-stage chronic kidney disease.

Material and methods. The retrospective, single-center, uncontrolled study involved 231 patients (132 M/99 W) aged 61.7 \pm 14.7 years with COVID-19 diagnosed. The SPSS software package was used for statistical analysis.

Results. $72(31.2 \%)$ of patients died, $68(94.4 \%)$ of them had ARDS as the main cause of death. Comparative analysis in groups with favorable and unfavorable outcomes of the disease showed that age (68.1 \pm 13.2 years vs $58.7 \pm 14.5$ years, $p<0.0001)$ and the comorbidity index $(8.8 \pm 2.2$ vs $6.2 \pm 2.6, p<0.0001)$ were significantly higher in those who have died compared to survivors. According to CT data, they were more likely to have $3^{\text {rd }}$ or $4^{\text {th }}$-degree lung damage $(72.2$ vs $36.5 \%, p<0.0001)$, and the minimum oxygen saturation index: $67.6 \pm 12.8$ and $87.8 \pm 10.9 \%$, respectively $(p<0.0001)$. Comorbidity index and the need for invasive ventilation were independent predictors of the fatal outcome of COVID-19. Early administration of monoclonal antibodies to IL-6 (in the first 3 days after hospitalization) in patients with a low prevalence of the pulmonary process (CT stage 1-2) was associated with a significantly lower frequency of fatal outcome.

Conclusions. SARS-CoV-2 infection in HD patients is characterized by a high rate of mortality. Predictors of severe disease in this population are comorbidity index and the need for invasive ventilation.

Funding. The study had no financial support.

Conflict of interests. The authors declare no conflict of interests.

Contribution. Research concept and design - Zubkin M.L., Frolova N.F., Kim I.G., Kryukov E.V., Chervinko V.I., Tomilina N.A.; collection and processing of material - Ushakova A.I., Usatyuk S.S., Artyukhina L.Yu. Iskhakov R.T., Varyasin V.V., Sinyavkin D.O., Diakova E.N., Loss K.E., Volodina E.V.; statistical data processing - Kim I.G.; text writing - Zubkin M.L., Kryukov E.V., Frolova N.F.; editing Zubkin M.L., Kim I.G., Kryukov E.V.; approval of the final version of the article - Zubkin M.L., Kryukov E.V., Frolova N.F., responsibility for the integrity of all parts of the article - Frolova N.F., Zubkin M.L.

For citation: Frolova N.F., Kim I.G., Ushakova A.I., Usatiuk S.S., Artyukhina L.Yu., Iskhakov R.T., Varyasin V.V., Sinyavkin D.0., Diakova E.N., Loss K.E., Chervinko V.I., Volodina E.V., Tomilina N.A., Kryukov E.V., Zubkin M.L. COVID-19 in hemodialysis patients. Infektsionnye bolezni: novosti, mneniya, obuchenie [Infectious Diseases: News, Opinions, Training]. 2021; 10 (1): 14-23. DOI: https://doi.org/10.33029/2305-3496-2021-10-1-14-23 (in Russian)

Received 19.11.2020. Accepted 28.12.2020.
Keywords: hemodialysis, COVID-19, SARS-CoV-2
$\mathrm{B}$ спышка новой коронавирусной инфекции (COVID-19) начавшаяся в 2019 г. в Китае, быстро достигла размеров пандемии. Как показала клиническая практика, почти у 80\% пациентов болезнь протекает бессимптомно или в виде легких респираторных проявлений $[1,2]$. Однако у $14 \%$ больных инфекция, вызванная этим вирусом (SARS(oV-2), приобретает тяжелое, а приблизительно у 6\% крайне тяжелое течение [3]. Большинство тяжелых случаев и летальных исходов выявлены у пожилых, а также у людей с хроническими заболеваниями [3]. Как правило, они проявляются развитием острого респираторного дистресс-синдрома (ОРДС), реже полиорганной недостаточностью или клинической картиной септического шока. Частое вовлечение в патологический процесс почек характеризуется широким диапазоном проявлений - от легкой протеинурии и гематурии до прогрессирующего острого повреждения почек, требующего применения заместительной почечной терапии (3ПТ) [4]. 
Пациенты, получающие 3ПТ в виде программного гемодиализа (ГД) по поводу терминальной стадии хронической болезни почек (тХБП), относятся к группе высокого риска инфицирования SARS-CoV-2, что обусловлено присутствием в диализных центрах значительного числа больных и медицинского персонала, некоторые из них могут оказаться вирусоносителями. Более того, больные на гемодиализе, как правило, имеют множество неблагоприятных факторов, осложняющих течение и отягощающих прогноз COVID-19. К таким факторам относят пожилой возраст, иммунодефицит различного генеза, в том числе обусловленный уремией, нарушения нутриционного статуса, а также наличие тяжелых сопутствующих заболеваний [5].

В публикациях из эпицентра новой коронавирусной инфекции (г. Ухань, Китай) сообщали о высокой частоте инфицирования SARS-CoV-2 в отделениях гемодиализа, достигавшей 16 и 14,3\% (по другим данным, от 1,1 до 19,6\%) $[6,7]$, а летальность составила 16,2 и 13,3\%, что существенно превышало этот показатель среди населения $[8,9]$.

Терапевтическая тактика у больных с тяжелым течением COVID-19 до сих пор является предметом дискуссии, хотя был достигнут определенный консенсус в отношении понимания этапности течения болезни. Во время первой фазы присутствуют эффекты, связанные с непосредственным патологическим воздействием вируса, и, соответственно, представляется целесообразным применение противовирусной терапии. Во второй, воспалительной, фазе болезни в условиях нарастания угрозы и появлении первых признаков «цитокинового шторма» рассматривается возможность иммуносупрессивной стратегии, в частности назначение глюкокортикоидов или иммунобиологических препаратов с антицитокиновым эффектом. Третья фаза течения оказывается угрожаемой в отношении развития тяжелых коагуляционных нарушений и/или полиорганной недостаточности $[10,11]$.

В связи с недостаточным опытом наблюдения за пациентами с ТХБП и COVID-19 в настоящее время очень мало данных о прогностических критериях тяжести течения и неблагоприятного исхода болезни, а также об оптимальных подходах к лечению в условиях применения ГД.

Цель исследования - анализ клинической картины, особенностей течения, факторов риска и возможностей терапии у больных COVID-19, получавших лечение программным гемодиализом.

\section{Материал и методы}

Под наблюдением с 1 апреля до 31 мая 2020 г. в ГБУЗ «ГКБ № 52» ДЗМ, перепрофилированном для оказания меди-

Таблица 1. Характеристика наблюдавшихся больных

\begin{tabular}{|c|c|}
\hline Показатель & Значение \\
\hline Количество больных (мужчины/женщины), $n$ & $231(132 / 99)$ \\
\hline Возраст, гоАы; $M \pm S D$ & $61,7 \pm 14,7$ \\
\hline $\begin{array}{l}\text { Средняя продолжительность лечения ГА, } \\
\text { мес, Ме (25\%; } 75 \%)\end{array}$ & $30,0(12 ; 68)$ \\
\hline $\begin{array}{l}\text { Время от появления первых симптомов } \\
\text { COVID-19 Ао госпитализации, AHи, } M \pm S D\end{array}$ & $5,8 \pm 5,0$ \\
\hline
\end{tabular}

цинской помощи пациентам, инфицированным SARS-CoV-2, находился 231 больной новой коронавирусной инфекцией, получавший лечение ГД по поводу тХБП. Исследование было одноцентровым, ретроспективным и неконтролируемым. Конечной точкой наблюдения считали выписку пациента из стационара или смерть. Больные, продолжавшие лечение на момент окончания исследования, из него были исключены.

Характеристика больных представлена в табл. 1 и 2. Наиболее многочисленными (около $30 \%$ ) оказались пациенты с сахарным диабетом (СД); большинство больных страдали СД 2-го типа. Далее следовали больные, у которых причинами развития тХБП были хронический гломерулонефрит и нефросклероз вследствие артериальной гипертензии (см. табл. 2).

Для подтверждения диагноза COVID-19 использовали полимеразную цепную реакцию (ПЦР) с целью выявления РНК вируса из материала мазков, полученных из носоглотки, и компьютерную томографию (КТ) органов грудной клетки. ПЦР проводили с помощью нескольких тест-систем: «Ампли Tест SARS-CoV-2» (ФГБУ «Центр стратегического планирования и управления медико-биологическими рисками здоровью» ФМБА России), «Поливир SARS-CoV-2 Express» (000 «Научно-производственная фирма “Литех"», Россия), «РеалБест PHK SARS-CoV-2» (ФБУН «Государственный научный центр вирусологии и биотехнологии "Вектор"», Россия). Результаты КТ оценивали согласно Временным методическим рекомендациям по профилактике, диагностике и лечению новой коронавирусной инфекции (COVID-19) [12]. ОРДС диагностировали на основании положений Берлинской классификации 2012 г. [13]. Индекс коморбидности (ИК) рассчитывали по Charlson [14].

В соответствии с имевшимися на момент госпитализации рекомендациями о подходах к лечению COVID-19 все пациенты получали противовирусную терапию лопинавиром (200 мг/сут), бустированным ритонавиром (50 мг/сут) в сочетании с гидроксихлорохином 200 мг/сут, антибиотиками и низкомолекулярным гепарином. Использовали несколько вариантов комбинаций антибиотиков: при неотягощенном течении заболевания - пенициллины с ингибиторами $\beta$-лактамазы + азитромицин \pm левофлоксацин; в более тяжелых случаях антибактериальная терапия была усилена антибиотиками других групп.

С целью профилактики тромботических осложнений на начальном этапе лечения больных COVID-19 использовали профилактические дозы низкомолекулярного гепарина. Однако по мере нарастания числа тромботических осложнений для поддержания адекватного гемостаза дозу антикоагулянтов увеличивали и в дальнейшем корректировали в соответствии с показателями коагулограммы и тромбоэластографиии.

При легкой форме течения COVID-19 пациенты получали иммунодепрессанты из группы ингибиторов янус-киназ (барицитиниб) и/или моноклональные антитела к рецепторам интерлейкина-6 (тоцилизумаб). В среднетяжелых и тяжелых случаях течения заболевания эти препараты применяли в комбинации с моноклональными антителами к интерлейкину-1 $\beta$ (канакинумаб) или к С5-компоненту ком- 
племента (экулизумаб). Выбор дозы иммунобиологических препаратов, особенно на первом этапе пандемии, проводили эмпирически. Это было связано с неопределенностью показаний к их назначению при тХБП и, соответственно, с недостаточным опытом такого лечения у данной категории больных. Это определило использование существенно более низких доз тоцилизумаба (3-6 мг/кг массы тела) по сравнению с рекомендуемыми фирмой-изготовителем для лечения ревматологических заболеваний - целевой нозологии для назначения указанных препаратов.

Все пациенты с признаками дыхательной недостаточности получали кислородную поддержку, а в случае ее неэффективности переводились на искусственную вентиляцию легких (ИВЛ).

Лечение ГД осуществляли в обычном режиме. Пациентам с тяжелым течением COVID-19, состояние которых требовало пребывания в отделении реанимации и интенсивной терапии, проводили продленную вено-венозную гемодиафильтрацию и реже - сеансы плазмообмена с введением до 2,5 л свежезамороженной плазмы.

Статистический анализ: при нормальном распределении непрерывных переменных рассчитывали средние значения, а в случаях их неправильного распределения - медиану. Сравнительный анализ средних показателей выполняли с помощью $t$-критерия Стьюдента. Категориальные переменные выражали абсолютными и относительными значениями и их различия оценивали методом $\chi^{2}$ Пирсона. При сравнении переменных значимыми считали различия при $p<0,05$. Для выявления факторов риска неблагоприятного исхода применяли однофакторный и многофакторный регрессионный анализ (модель Сох). При статистической обработке данных использовали пакет программ SPSS (версия 22).

\section{Результаты и обсужкение}

У $198(85,7 \%)$ больных инфицирование SARS-CoV-2 было подтверждено результатами ПЦР, а у остальных - характерной картиной, определяемой при КТ органов грудной клетки.

Средняя продолжительность пребывания пациентов в стационаре составила $12,2 \pm 6,6$ дня. Наиболее часто у больных COVID-19, получавших лечение ГД, регистрировали лихорадку (96\%), выраженную слабость (95\%), одышку (70,6\%) и кашель (53,3\%). По данным КТ органов грудной клетки почти у половины больных (47,6\%) были выявлены тяжелая (3-я) или крайне тяжелая (4-я) степень поражения легких (табл. 3). Уровень сатурации кислорода в крови $\left(\mathrm{SpO}_{2}\right)$ во время болезни был ниже нормы у подавляющего большин-

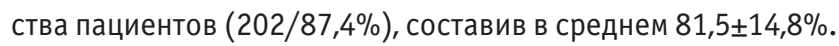
При $\mathrm{SpO}_{2} \leq 92 \%(n=159)$ все они получали респираторную поддержку, в том числе 10 (6,3\%) - неинвазивную ИВЛ, а 60 $(37,7 \%)-$ ИВЛ.

За время наблюдения умерли 72 (31,2\%) пациента. Наиболее частой причиной летального исхода оказался ОРДС, который был диагностирован в $63(87,5 \%)$ случаях. При этом у 30 их них течение болезни было отягощено такими тяжелыми патологическими состояниями, как сердечная или полиорганная недостаточность, тромбоэмболия легоч-
Таблица 2. Причины развития терминальной стадии хронической болезни почек

\begin{tabular}{|l|c|c|}
\hline \multirow{2}{*}{\multicolumn{1}{|c|}{ Нозология }} & \multicolumn{2}{|c|}{ Число больных } \\
\cline { 2 - 3 } & абс. & $\%$ \\
\hline Сахарный Аиабет & 69 & 29,9 \\
(1-го типа/2-го типа) & $(9 / 60)$ & $(13,1 / 86,9)$ \\
\hline Хронический гломерулонефрит & 41 & 17,7 \\
\hline Гипертоническая болезнь & 24 & 10,4 \\
\hline Хронический пиелонефрит & 10 & 9,1 \\
\hline Нефропатия неясной этиологии & 17 & 7,4 \\
\hline Поликистоз почек & 13 & 5,6 \\
\hline Ишемическая нефропатия & 10 & 4,3 \\
\hline Мочекаменная болезнь & 10 & 4,3 \\
\hline Аномалия развития мочевой системы & 8 & 3,5 \\
\hline Миеломная болезнь & 5 & 2,2 \\
\hline Системные васкулиты & 5 & 2,2 \\
\hline Подагра & 3 & 1,3 \\
\hline Тромботическая микроангиопатия & 2 & 0,9 \\
\hline Синдром Альпорта & 1 & 0,4 \\
\hline Амилоиноз & 1 & 0,4 \\
\hline Псориаз & 1 & 0,4 \\
\hline Всего & 231 & 100 \\
\hline
\end{tabular}

ной артерии, кровотечения, тромботическая микроангиопатия, сепсис. Еще у 9 больных без признаков ОРДС причиной смерти стали осложнения со стороны сердечно-сосудистой системы и сепсис.

Летальный исход при COVID-19 у больных на ГД был ассоциирован с более тяжелым и распространенным поражением легких (КТ-3 и КТ-4), которое диагностировали достоверно чаще по сравнению с выжившими больными (72,2 vs $36,5 \%, p<0,01 ;$ см. табл. 3). Средний показатель сатурации в этих группах был $66,9 \pm 11,1$ и $87,8 \pm 10,9 \%$ соответственно $(p<0,05)$.

Результаты сравнительного анализа клинических данных в группах больных с благоприятным и неблагоприятным исходом представлены на рисунке.

Умершие пациенты были старше $(p<0,05)$ и имели достоверно более высокий ИК, в том числе за счет конкурирующих сердечно-сосудистых заболеваний (СС3), цереброваскулярной болезни (ЦВБ), а также хронической обструктивной болезни легких (ХОБЛ). Частота СД и индекс массы тела

Таблица 3. Распределение больных по степени тяжести поражения ^егких, по Аанным компьютерной томографии (КТ)

\begin{tabular}{c|c|c|c|c}
$\begin{array}{c}\text { Степень } \\
\text { поражения } \\
\text { легких по } \\
\text { Аанным KT }\end{array}$ & $\begin{array}{c}\text { В целом } \\
\text { при посту- } \\
\text { плении, } \\
n(\%)\end{array}$ & $\begin{array}{c}\text { 1-я группа } \\
\text { (выжив- } \\
\text { шие), } \\
n(\%)\end{array}$ & $\begin{array}{c}\text { 2-я группа } \\
\text { (умер- } \\
\text { шие), } \\
n(\%)\end{array}$ & \\
\hline KT-1 & $23(10)$ & $17(10,7)$ & $6(8,3)$ & 0,17 \\
\hline KT-2 & $98(42,4)$ & $84(52,8)$ & $14(19,4)$ & 0,01 \\
\hline KT-3 & $91(39,4)$ & $56(35,2)$ & $35(48,6)$ & 0,14 \\
\hline KT-4 & $19(8,2)$ & $2(1,3)$ & $17(23,6)$ & 0,01 \\
\hline Bсего & 231 & 159 & 72 & \\
\hline
\end{tabular}

* - проведено сравнение между 1-й и 2-й группами. 


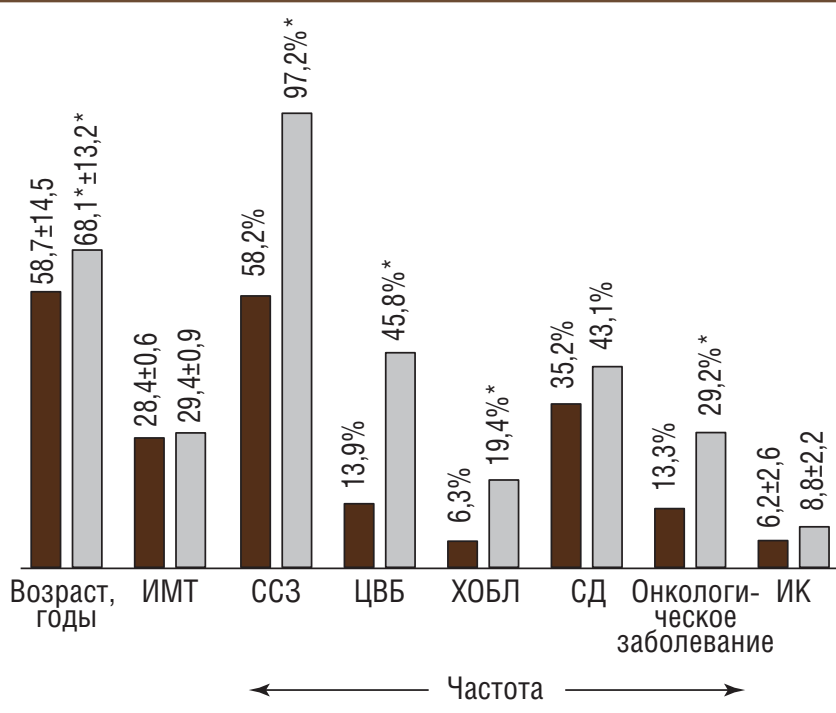

* $-p<0,05 \square$ 1-я группа $(n=159) \quad \square$ 2-я группа $(n=72)$

Клиническая характеристика больных с благоприятным (1-я группа) и неблагоприятным (2-я группа) исходом болезни Расшифровка аббревиатур дана в тексте.

среди них были лишь незначительно и статистически недостоверно выше (43,1 против 35,2\% и 29,4 40,9 против $28,4 \pm 0,6$ кг/м² соответственно), чем у больных с благополучным течением COVID-19.

Данные лабораторных исследований крови пациентов представлены в табл. 4. Обращали на себя внимание сниженные уровни гемоглобина, лимфоцитов и альбумина, а также высокие показатели С-реактивного белка (СРБ), ферритина, аланин- (АЛТ) и аспартатаминотрансферазы (АСТ), $\gamma$-глутамилтранспептидазы (ГГТ), глюкозы, лактатдегидрогенезы (ЛДГ), фибриногена и D-димера. Низкий уровень лейкоцитов наблюдался, как правило, в дебюте болезни с тенденцией к превышению нормальных значений в случае развития септических осложнений.
Были установлены значимые различия лабораторных параметров у диализных больных с COVID-19 в зависимости от исхода заболевания (см. табл. 4).

С неблагоприятным исходом были ассоциированы как лейкоцитоз, так и лейкопения, лимфопения, тромбоцитопения, гипоальбуминемия. У умерших больных определяли более высокие значения СРБ, D-димера, АСТ, ГГТ, ЩФ, ЛДГ и глюкозы.

Не удалось установить связь летального исхода с поздним оказанием квалифицированной медицинской помощи: сроки госпитализации от начала болезни в группах умерших

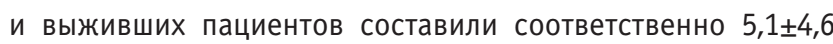
и 6,1 4,8 дня. Сравниваемые группы также оказались сопоставимы по продолжительности лечения ГД: $30(12 ; 65)$ и 29,5 $(12 ; 71,5)$ мес соответственно.

Оценка связи вариантов терапии COVID-19 с исходом заболевания у больных, получавших лечение ГД, показала, что летальность пациентов не зависела от количества антибактериальных препаратов в использованных схемах терапии: защищенные пенициллины с азитромицином или левофлоксацином; защищенные пенициллины с азитромицином и левофлоксацином; защищенные пенициллины с азитромицином и/или левофлоксацином в комбинации с антибиотиками из других групп.

При степени поражения легких КТ 1-2 большая часть пациентов получали 2 антибактериальных препарата (83 по сравнению с 38 больными, которым были назначены 3 антибиотика и более). Независимо от схемы антибактериальной терапии число летальных исходов оказалось примерно одинаковым в этих подгруппах - 12 и 8 соответственно. При степени поражения легких КТ 3-4 большая часть пациентов также получали 2 антибиотика (74 против 36 - на 3 антибактериальных препаратах и более). Число летальных исходов также было одинаковым (26 больных) и не зависело от схемы антибактериальной терапии, однако умерших с этой степенью поражения легких было больше (52 человека), чем в подгруппе с КТ 1-2 (20 пациентов).

Иммунобиологические препараты в составе патогенетической терапии применяли у 167 (72,3\%) из 231 пациента.

Таблица 4. ^абораторные показатели пациентов с благоприятным и неблагоприятным исходом болезни

\begin{tabular}{|c|c|c|c|c|}
\hline Показатель & $\begin{array}{l}\text { В целом, } M \pm S D \\
M е(25 \% ; 75 \%)\end{array}$ & $\begin{array}{c}\text { 1-я группа, } M \pm S D \\
M e(25 \% ; 75 \%)\end{array}$ & $\begin{array}{c}\text { 2-я группа, } M \pm S D \\
M e(25 \% ; 75 \%)\end{array}$ & $p^{*}$ \\
\hline Гемоглобин, г/^ & $87,3 \pm 17,2$ & $87,9 \pm 17,0$ & $85,9 \pm 17,8$ & 0,4 \\
\hline лейкоциты, ×109/^, максимальный уровень & $10,0 \pm 6,3$ & $7,8 \pm 3,6$ & $17,7 \pm 9,47$ & 0,001 \\
\hline лейкоциты, ×109/^, минимальный уровень & $3,7 \pm 2,1$ & $4,4 \pm 2,9$ & $3,3 \pm 1,6$ & 0,001 \\
\hline Аимфоциты, ×109/^ & $0,6 \pm 0,5$ & $0,7 \pm 0,5$ & $0,5 \pm 0,4$ & 0,001 \\
\hline Тромбоциты, ×109/^ & $174,3 \pm 126,4$ & $188,9 \pm 135,9$ & $142,1 \pm 95,7$ & 0,009 \\
\hline Ферритин, мкг/^ & $579,8 \pm 214,9$ & $564,8 \pm 218,3$ & $621,3 \pm 201,8$ & 0,08 \\
\hline СРБ, Мг/^ & $134,4 \pm 98,1$ & $110,3 \pm 88,6$ & $187,8 \pm 98,3$ & 0,001 \\
\hline Альбумин, г/^ & $29,9 \pm 4,7$ & $31,2 \pm 4,2$ & $27,2 \pm 4,5$ & 0,001 \\
\hline ГАюкоза, ммоль/^ & $8,1 \pm 5,2$ & $7,2 \pm 4,9$ & $9,4+5,5$ & 0,014 \\
\hline$\Lambda А Г, M E / \Lambda$ & $506,1 \pm 347,5$ & $395,7 \pm 163,4$ & $749,9 \pm 494,8$ & 0,001 \\
\hline Фибриноген, г/^ & $6,9 \pm 1,8$ & $7,0 \pm 1,7$ & $6,9 \pm 1,9$ & 0,5 \\
\hline $\mathrm{A} \wedge \mathrm{T}, \mathrm{ME} / \wedge$ & $24,7(15,1 ; 44,8)$ & $24,7(14,9 ; 46,3)$ & $23,5(15,0 ; 40,4)$ & 0,06 \\
\hline $\mathrm{ACT}, \mathrm{ME} / \wedge$ & $41,9(28,3 ; 68,6)$ & $39,0(26,6 ; 60,7)$ & $50,2(29,9 ; 119,7)$ & 0,001 \\
\hline 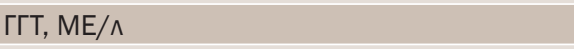 & $45,3(26,0 ; 92,1)$ & $42,9(23,9 ; 78,8)$ & $56,7(26,7 ; 101,6)$ & 0,02 \\
\hline D-димер, мкг/^ & $742,0(413,8 ; 607,0)$ & $624,0(381,0 ; 950,0)$ & $1291,0(743 ; 3258)$ & 0,001 \\
\hline
\end{tabular}

Примечание. * - проведено сравнение между 1-й и 2-й группами; здесь и в табл. 5: расшифровка аббревиатур дана в тексте. 
Таблица 5. Предикторы неблагоприятного исхода COVID-19 у больных, получавших лечение гемодиализом (многофакторный регрессионный анализ, модель Сох)

\begin{tabular}{|c|c|c|c|}
\hline Параметр & Коэффициент риска (соотношение шансов) & 95\% доверительный интервал & $p$ \\
\hline ИК & 1,2 & $1,0-1,3$ & 0,008 \\
\hline ИВ $\Lambda$ & 11,2 & $5,1-24,2$ & 0,0001 \\
\hline
\end{tabular}

В виде монотерапии тоцилизумаб или барицитиниб был назначен 100 больным, остальные 67 получали одновременно оба препарата (51 пациент) или в сочетании с канакинумабом или экулизумабом (16 человек). В подгруппе монотерапии умерли 25 (25,0\%) больных, а в подгруппе с большим числом компонентов - 21 (31,3\%) больной. Статистически значимых различий не выявлено.

Используемая доза тоцилизумаба не влияла на исход заболевания $(3,3 \pm 1,8$ мг/кг - с благоприятным исходом и 2,8 $\pm 1,3$ мг/кг - с неблагоприятным исходом). В то же время выявлена статистически достоверная связь между назначением тоцилизумаба в первые 3 сут госпитализации с благоприятным исходом инфекции у больных с КТ 1-2. Из 29 пациентов со степенью КТ 1-2, получавших тоцилизумаб в первые 3 сут после госпитализации, умер 1 (3,4\%), а из 20 больных, которым назначали этот же препарат в более поздние сроки, умерли 6 (24,1\%) пациентов, $p<0,04$. У больных с КТ 3-4 обнаружена лишь тенденция к снижению числа неблагоприятных исходов болезни при назначении тоцилизумаба в первые 3 сут после госпитализации из 38 пациентов умерли 14 (36,8\%). В подгруппе пациентов, которым этот препарат назначили позднее, умерли $11(47,8 \%)$ из 23 больных ( $p>0,05)$.

Следует отметить, что течение COVID-19 сопровождалось развитием коагуляционных осложнений. Тромбозы различной локализации (преимущественно тромбоэмболия ветвей легочной артерии и артериовенозной фистулы) были диагностированы у 22 (9,5\%) больных, что потребовало увеличения дозы антикоагулянтных препаратов; также возросло число случаев спонтанного кровотечения (преобладали гематомы мягких тканей). Их наблюдали у 16 (6,9\%) пациентов.

При использовании многофакторной модели Сох независимыми предикторами неблагоприятного исхода COVID-19 у больных, получавших лечение ГД, оказались ИК и применение ИВЛ (табл. 5).

Новая коронавирусная инфекция, вызванная SARS-CoV-2, пока еще малоизучена. Однако накопленные за этот короткий период наблюдения позволяют утверждать, что наибольшую угрозу она представляет для людей пожилого возраста, а также для пациентов с тяжелыми хроническими заболеваниями [15]. По данным Итальянского института здоровья, среди умерших от COVID-19, только 1\% не имели сопутствующих болезней, тогда как $26 \%$ страдали по крайней мере 1 ; $26 \%-2$, а $47 \%$ - 3 сопутствующими заболеваниями. Наиболее частыми из них были артериальная гипертензия (70\%), другая сердечно-сосудистая патология (38,5\%), СД (31,7\%), ХБП $(23,1 \%)$, ХОБЛ $(18,1 \%)$, наличие активного онкологического процесса в предшествующие 5 лет до инфицирования SARS-CoV-2 (16,8\%) и ожирение (10\%) [16]. 0 высокой частоте сопутствующих болезней почек при COVID-19 также сообщалось в ряде других публикаций. В целом и развитие острого повреждения почек, и наличие ХБП были признаны независимыми факторами риска неблагоприятного исхода при COVID-19 [17-19]. В частности, было установлено, что среди умерших пациентов более 20\% имели ХБП [20], которая, по данным одного из метаанализов, оказалась связана не только с повышенным риском заражения, но и с более тяжелым течением COVID-19 [21].

В то же время информация о частоте инфицирования, характере течения и прогнозе COVID-19 у больных с терминальной стадией ХБП, получающих лечение ГД, достаточно противоречива. Несмотря на отдельные утверждения об относительно небольшой распространенности этой инфекции и ее благоприятном прогнозе среди пациентов, получающих лечение диализом [22, 23], все же преобладают представления о более высокой предрасположенности гемодиализных больных к заражению SARS-CoV-2 по сравнению с людьми без тХБП [24, 25]. Полагают, что у пациентов на ГД заболевание COVID-19 приобретает более тяжелое течение по сравнению с больными, имевшими нормальную почечную функцию. Так, если уровень летальности среди населения колеблется в пределах от 0,3-1,4 до 8-10\% [26-29], то, согласно данным регистра Европейской почечной ассоциации - Европейской ассоциации диализа и трансплантации (ERA-EDTA) [30], за весну-осень 2020 г. среди 5596 диализных больных летальный исход наблюдали у 1331 (23,8\%). По материалам, представленным из разных регионов Италии, летальность диализных пациентов колебалась в пределах от 25 до 40\% [27, 31, 32]. В то же время в нескольких центрах Китая она не превышала 13-16\% [33]. Наиболее близкой к полученным в данном исследовании показателям $(31,2 \%)$ оказалась частота летальных исходов в одном из диализных центров Испании, где 36 (12,8\%) из 282 больных были инфицированы SARS-CoV-2, а доля умерших составила $30,5 \%$ [34].

Причиной смерти большинства пациентов в проведенном исследовании оказался ОРДС (у 63 из 72 больных; 87,5\% случаев) нередко в сочетании с другой патологией, что сопоставимо с данными в итальянской когорте [27, 31, 32], однако они контрастируют с выборкой из г. Ухань (Китай), в которой основной причиной смерти были названы ССЗ/ осложнения [8]. В данном исследовании не удалось установить связи между неблагоприятным исходом COVID-19 с длительностью лечения ГД и сроком госпитализации после появления первых признаков коронавирусной инфекции. В то же время, как отмечают многие другие исследователи $[24,35]$, была подтверждена неблагоприятная прогностическая роль тяжелой сопутствующей патологии, в частности СС3, цереброваскулярной болезни и ХОБЛ, что нашло отражение в более высоком ИК, выявленном у больных с летальным исходом. В отличие от ряда авторов [36-38], в данной работе, как и в исследовании F. Xiong и соавт. [24], 
не выявлено связи избыточной массы тела и наличия СД с исходом болезни у диализных пациентов. Тем не менее более высокий уровень гликемии значимо чаще определяли у умерших больных $(p<0,05)$, что, по-видимому, свидетельствует о прогностической роли декомпенсации этого заболевания. С неблагоприятным прогнозом течения болезни были ассоциированы и другие клинические факторы: лейкоцитоз или лейкопения, лимфопения, гипоальбуминемия, высокие показатели СРБ, D-димера, активности ЛДГ, а также степень поражения легких КТ 3-4 и использование ИВЛ. При этом независимыми предикторами летального исхода, по данным регрессионного многофакторного анализа, оказались высокий ИК и необходимость применения ИВЛ. Полученные результаты полностью согласуются с выводами большой группы исследователей, анализировавших такого рода взаимосвязи как для больных на ГД, так и среди населения $[16,24,34,39]$.

Лечение COVID-19, которое получали пациенты в отделении гемодиализа ГБУЗ «ГКБ № 52» ДЗМ, проводили в полном соответствии с международными, в том числе В03, рекомендациями, существовавшими на ранней стадии развития пандемии. Дозы антикоагулянтов и особенно препаратов моноклональных антител к рецептору ИЛ-6, применявшиеся на том этапе, в настоящее время не могут рассматриваться в качестве адекватных. Такая оценка в полной мере относится и к подходам по назначению антибактериальных препаратов.
Не установлена четкая связь терапии COVID-19 с исходом болезни. Однако была выявлена тенденция, не подтвержденная статистически, свидетельствующая о возрастающей частоте неблагоприятных исходов по мере увеличения числа применявшихся антибактериальных и иммунобиологических препаратов. Такая тактика была проявлением «терапии отчаяния» у наиболее тяжелых, резистентных к стандартному лечению больных, однако при этом нельзя исключить и негативную роль полипрагмазии.

В то же время хотелось бы привлечь внимание к обнадеживающему результату раннего назначения (в первые 3 сут после госпитализации) препаратов моноклональных антител к рецепторам ИЛ-6 у больных с небольшим объемом поражения легких (КТ 1-2). Вероятно, назначение этих препаратов в начальной стадии болезни при малой распространенности вирусного поражения легких может существенно повысить эффективность терапии. Для усовершенствования методов лечения COVID-19, несомненно, необходимы дальнейшие, предпочтительно проспективные исследования.

Таким образом, COVID-19 у значительного числа больных с терминальной стадией ХБП, получающих лечение ГД, отличается тяжелым течением и неблагоприятным прогнозом. В связи с этим существует необходимость поиска эффективных и безопасных методов профилактики и лечения в группах повышенного риска инфицирования и тяжелого течения COVID-19.

\section{CBEАEНИЯ ОБ АВTOPAX}

Фролова Надия Фяатовна (Nadiya F. Frolova) - кандидат медицинских наук, заместитель главного врача по нефрологической помощи ГБУЗ «ГКБ № 52» ДЗМ, Москва, Российская Федерация

E-mail: nadiya.frolova@yandex.ru

Ким Ирина Гиховна (Irina G. Kim) - кандидат медицинских наук, ведущий научный сотрудник ФБУН МНИИЭМ им. Г.Н. Габричевского Роспотребнадзора, Москва, Российская Федерация

E-mail: kig21@rambler.ru

Ушакова Анжела Ильинична (Anzhela I. Ushakova) - заведующий отделением гемодиализа ГБУз «ГКБ № 52» ДЗМ, Москва, Российская Федерация

E-mail: anzhela_52@inbox.ru

Усатюк Сергей Сергеевич (Sergei S. Usatiuk) - заведующий нефрологическим отделением ГБУЗ «ГКБ № 52» ДЗМ, Москва, Российская Федерация

E-mail: usatuk-doc@mail.ru

Артюхина Людмила Юрьевна (Lyudmila Yu. Artyukhina) - кандидат медицинских наук, заведующий отделением патологии трансплантированной почки ГБУЗ «ГКБ № 52» ДЗМ, Москва, Российская Федерация

E-mail: arlyu-1404@yandex.ru

https://orcid.org/0000-0003-3353-1636

Исхаков Рустам Тимурович (Rustam T. Iskhakov) - заведующий отделением реанимации и интенсивной терапии для больных нефрологического профиля ГБУЗ «ГКБ № 52» ДЗМ, Москва, Российская Федерация

E-mail: stamius@yandex.ru

Варясин Валерий Викторович (Valeriy V. Varyasin) - кандидат медицинских наук, заведующий патологоанатомическим отделением ГБУЗ «ГКБ № 52» ДЗМ, Москва, Российская Федерация

E-mail:pa052@mail.ru

https://orcid.org/0000-0003-1136-6203

Синявкин Дмитрий Ованесович (Dmitry 0. Sinyavkin) - заведующий клинико-диагностической лабораторией ГБУз «ГКБ № 52» Д3М, Москва, Российская Федерация

E-mail:dsmaus@mail.ru

Дьякова Екатерина Николаевна (Ekaterina N. Diakova) - врач нефрологического отделения ГБУз «ГКБ № 52» ДЗМ, Москва, Российская Федерация

E-mail: katerina-diakova@mail.ru 
Лосс Карина Эдуардовна (Karina E. Loss) - врач отделения реанимации и интенсивной терапии для больных нефрологического профиля ГБУЗ «ГКБ № 52» ДЗМ, Москва, Российская Федерация

E-mail: karina1512@mail.ru

Червинко Валерий Иванович (Valeriy I. Chervinko) - кандидат медицинских наук, доцент кафедры терапии неотложных состояний филиала Военно-медицинской академии им. С.М. Кирова Минобороны России, Москва, Российская Федерация

E-mail:dok534@yandex.ru

https://orcid.org/0000-0003-1051-2897

Володина Елизавета Владимировна (Elizaveta V. Volodina) - научный сотрудник ФБУН МНИИЭМ им. Г.Н. Габричевского Роспотребнадзора, Москва, Российская Федерация

E-mail:vol.e.v@yandex.ru

Томилина Наталья Аркадьевна (Natalia A. Tomilina) - доктор медицинских наук, профессор, заведующий кафедрой нефрологии факультета дополнительного профессионального образования ФГБОУ Во МГМСУ им. А.И. Евдокимова Минздрава России, Москва, Российская Федерация

E-mail: natomilina@yandex.ru

Крюков Евгений Владимирович (Evgeniy V. Kryukov) - член-корреспондент РАН, доктор медицинских наук, профессор, начальник ФГБУ «ГВКГ им. Н.Н. Бурденко» Минобороны России, Москва, Российская Федерация

E-mail: evgeniy.md@mail.ru

https://orcid.org/0000-0002-8396-1936

Зубкин Михаил Леонидович (Mikhail L. Zubkin) - доктор медицинских наук, профессор, руководитель клинико-диагностического отдела ФБУН МНИИЭМ им. Г.Н. Габричевского Роспотребнадзора, Москва, Российская Федерация

E-mail:m-zubkin@yandex.ru

https://orcid.org/0000-0001-5271-1902

\section{ヘИТЕРАТУРА}

1. Bai Y., Yao L., Wei T., Tian F., Jin D.Y., Chen L. et al. Presumed asymptomatic carrier transmission of COVID-19 // JAMA. 2020. Vol. 323, N 14. P. $1406-1407$.

2. Li R., Pei S., Chen B., Song Y., Zhang T., Yang W. et al. Substantial undocumented infection facilitates the rapid dissemination of novel coronavirus (SARS-CoV2) // Science. 2020. Vol. 368, N 6490. P. 489-493.

3. Guan W.J., Ni Z.Y., Hu Y., Liang W.H., Ou C.Q., He J.X. et al. Clinical characteristics of coronavirus disease 2019 in China // N. Engl. J. Med. 2020. Vol. 382, N 18. P. 1708-1720.

4. Perico L., Benigni A., Remuzzi G. Should COVID-19 concern nephrologists? Why and to what extent? The emerging impasse of angiotensin blockad // Nephron. 2020. Vol. 144, N 5. P. 213-221.

5. Ronco C., Reis T., Husain-Syed F. Management of acute kidney injury in patients with COVID-19 // Lancet Respir. Med. 2020. Vol. 8, N 7. P. 738-742.

6. Arslan H., Musabak U., Soy E.H.A., Azap O.K., Sayin B., Akcay S. et al. Incidence and immunologic analysis of coronavirus disease (COVID-19) in hemodialysis patients: a single-center experience // Exp. Clin. Transplant. 2020. Vol. 18, N 3. P. 275-283.

7. Corbett R.W., Blakey S., Nitsch D., Loucaidou M., McLean A., Duncan N. et al. Epidemiology of COVID-19 in an Urban Dialysis Center // J. Am. Soc. Nephrol. 2020. Vol. 31, N 8. P. 1815-1823.

8. Ma Y., Diao B., Lv X., Zhu J., Liang W., Liu L. et al. 2019 nove coronavirus disease in hemodialysis (HD) patients: report from one HD center in Wuhan China // medRxiv. 2020. DOI: https://doi.org/10.1101/ 2020.02.24.20027201

9. Li C., Yonglong M., Can T., Dongdong M., Sheng W., Haifeng L. et al. An Analysis on the clinical features of MHD patients with coronavirus disease 2019: a single center study. 2020. DOI: https://doi.org/10.21203/ rs.3.rs-18043/v1 URL: https://www.researchsquare.com/article/rs18043/v1

10. Alberici F., Delbarba E., Manenti C., Econimo L., Valerio F., Pola A et al. Management of patients on dialysis and with kidney transplant during SARS-COV-2 (COVID-19) pandemic in Brescia, Italy // Kidney Int. Rep. 2020. Vol. 5, N 5. P. 580-585.

11. Bonny V., Maillard A., Mousseaux C., Plaçais L., Richier Q. COVID19: Pathogenesis of a multi-faceted disease // Rev. Med. Interne. 2020. Vol. 41, N 6. P. 375-389.

12. Временные методические рекоменаации "Профилактика, Аиагностика и мечение новой коронавирусной инфекции (COVID-19)" Версия 8.1 (1 октября 2020 г.). Москва, 2020.
13. Ranieri V.M., Rubenfeld G.D., Thompson B.T., Ferguson N.D., Caldwell E., Fan E. et al. Acute respiratory distress syndrome: the Berlin Definition // JAMA. 2012. Vol. 307, N 23. P. 2526-2533.

14. Hemmelgarn B.R., Manns B.J., Quan H., Ghali W.A. Adapting the Charlson Comorbidity Index for use in patients with ESRD // Am. J. Kidney Dis. 2003. Vol. 42, N 1. P. 125-132.

15. Zhang Y.; The Novel Coronavirus Pneumonia Emergency Response Epidemiology Team. The epidemiological characteristics of an outbreak of 2019 novel coronavirus diseases (COVID-19) - China, 2020 // China CDC Weekly. 2020. Vol. 2. P. 113-122.

16. Gagliardi I., Patella G., Michael A., Serra R., Provenzano M., Andreucci M. COVID-19 and the kidney: from epidemiology to clinical practice // J. Clin. Med. 2020. Vol. 9, N 8. P. 2506.

17. Cheng Y., Luo R., Wang K., Zhang M., Wang Z., Dong L. et al. Kidney disease is associated with in-hospital death of patients with COVID-19 // Kidney Int. 2020. Vol. 97, N 5. P. 829-838.

18. Wang L., Li X., Chen H., Yan S., Li D., Li Y. et al. Coronavirus disease 19 infection does not result in acute kidney injury: an analysis of 116 hospitalized patients from Wuhan, China // Am. J. Nephrol. 2020. Vol. 51, N 5. P. $343-348$.

19. Zhang J., Litvinova M., Wang W., Wang Y., Deng X., Chen X. et al. Evolving epidemiology and transmission dynamics of coronavirus disease 2019 outside Hubei province, China: a descriptive and modelling study // Lancet Infect. Dis. 2020. Vol. 20, N 7. P. 793-802.

20. Boraschi P. COVID-19 Pulmonary involvement: is really an interstitial pneumonia? // Acad. Radiol. 2020. Vol. 27, N 6. P. 900.

21. Henry B.M., Lippi G. Chronic kidney disease is associated with severe coronavirus disease 2019 (COVID-19) infection // Int. Urol. Nephrol. 2020. Vol. 52, N 6. P. 1193-1194.

22. Wang R., Liao C., He H., Hu C., Wei Z., Hong Z. et al. COVID-19 in hemodialysis patients: a report of 5 cases // Am. J. Kidney Dis. 2020. Vol. 76, N 1. P. 141-146.

23. Hande A., Ugur M., Ebru H.A.S., Ozlem K.A., Burak S., Sule A. et al. Incidence and immunologic analysis of coronavirus disease (COVID19) in hemodialysis patients: a single-center experience // Exp. Clin. Transplant. 2020. Vol. 18, N 3. P. 275-283.

24. Xiong F., Tang H., Liu L., Tu C., Tian J., Lei C. et al. Clinical characteristics of and medical interventions for COVID-19 in hemodialysis patients in Wuhan, China // J. Am. Soc. Nephrol. 2020. Vol. 31, N 7. P. 1387-1397.

25. Rombola G., Brunini F. COVID-19 and dialysis: why we should be worried // J. Nephrol. 2020. Vol. 33, N 3. P. 401-403. 
26. Guan W.J., Ni Z.Y., Hu Y., Liang W.H., Ou C.Q., He J.X. et al. Clinica characteristics of coronavirus disease 2019 in China // N. Engl. J. Med. 2020. Vol. 392, N 18. P. 1708-1720.

27. Grasselli G., Zangrillo A., Zanella A., Antonelli M., Cabrini L., Castelli A. et al. COVID-19 Lombardy ICU Network. Baseline characteristics and outcomes of 1591 patients infected with SARS-CoV-2 admitted to ICUs of the Lombardy Region, Italy // JAMA. 2020. Vol. 323, N 16. P. 1574-1581.

28. Yi Y., Lagniton P.N.P., Ye S., Li E., Xu R.H. COVID-19: what has been learned and to be learned about the novel coronavirus disease // Int. J. Biol. Sci. 2020. Vol. 16, N 10. P. 1753-1766.

29. Madjid M., Safavi-Naeini P., Solomon S.D., Vardeny O. Potential effects of coronaviruses on the cardiovascular system: a review // JAMA Cardiol. 2020. Vol. 5, N 7. P. 831-840.

30. COVID-19 Registro ERA-EDTA. URL: https://www.era-edta.org/en/ registry/covid-19 (date of access 3 August, 2020)

31. Alberici F., Delbarba E., Manenti C., Econimo L., Valerio F., Pola A. et al. Management of patients on dialysis and with kidney transplantation during the SARS-CoV-2 (COVID-19) pandemic in Brescia, Italy // Kidney Int. Rep. 2020. Vol. 5, N 5. P. 580-585.

32. Scarpioni R., Manini A., Valsania T., Amicis S.D., Albertazzi V., Melfa L. et al. COVID-19 and its impact on nephropathic patients: the experience at Ospedale "Guglielmo da Saliceto" in Piacenza // G. Ital. Nefrol. 2020. Vol. 37, N 2. P. 1-5.
33. Ma Y., Diao B., Lv X., Zhu J., Liang W., Liu L. et al. 2019 nove coronavirus disease in hemodialysis (HD) patients: report from one HD center in Wuhan China // medRxiv. 2020. DOl: https://doi.org/10.1101/ 2020.02.24.20027201 (date of access June 17, 2020)

34. GoicoecheaM.,LuisCamaraA.S.,MacíasN.,MoralesA.M.,RojasA.G., Bascunana A. et al. COVID-19: clinical course and outcomes of 36 hemodialysis patients in Spain // Kidney Int. 2020. Vol. 98, N 1. P. 27-34.

35. Manganaro M., Baldovino S. First considerations on the SARSCoV-2 epidemic in the Dialysis Units of Piedmont and Aosta Valley, Northern Italy // J. Nephrol. 2020. Vol. 33, N 3. P. 393-395.

36. Singh A.K., Gupta R., Ghosh A., Misra A. Diabetes in COVID-19: prevalence, pathophysiology, prognosis and practical considerations // Diabetes Metab. Syndr. 2020. Vol. 14, N 4. P. 303-310.

37. Scheen A.J. Obesity and risk of severe COVID-19 // Rev. Med. Suisse. 2020. Vol. 16, N 695. P. 1115-1119.

38. Li X., Wang L., Yan S., Yang F., Xiang L., Zhu J. et al. Clinical characteristics of 25 death cases with COVID-19: a retrospective review of medical records in a single medical center, Wuhan, China // Int. J. Infect. Dis. 2020 Vol. 94. P. $128-132$

39. Zhou F., Yu T., Du R., Fan G., Liu Y., Liuet Z. et al. Clinical course and risk factors for mortality of adult inpatients with COVID-19 in Wuhan China: a retrospective cohort study // Lancet 2020. Vol. 395, N 10229. P. 1054-1062.

\section{REFERENCES}

1. Bai Y., Yao L., Wei T., Tian F., Jin D.Y., Chen L., et al. Presumed asymptomatic carrier transmission of COVID-19. JAMA. 2020; 323 (14): 1406-7.

2. Li R., Pei S., Chen B., Song Y., Zhang T., Yang W., et al. Substantia undocumented infection facilitates the rapid dissemination of novel coronavirus (SARS-CoV2). Science. 2020; 368 (6490): 489-93.

3. Guan W.J., Ni Z.Y., Hu Y., Liang W.H., Ou C.Q., He J.X., et al. Clinical characteristics of coronavirus disease 2019 in China. N Engl J Med. 2020; 382 (18): 1708-20.

4. Perico L., Benigni A., Remuzzi G. Should COVID-19 concern nephrologists? Why and to what extent? The emerging impasse of angiotensin blockad. Nephron. 2020; 144 (5): 213-21.

5. Ronco C., Reis T., Husain-Syed F. Management of acute kidney injury in patients with COVID-19. Lancet Respir Med. 2020; 8 (7): 738-42.

6. Arslan H., Musabak U., Soy E.H.A., Azap O.K., Sayin B., Akcay S., et al. Incidence and immunologic analysis of coronavirus disease (COVID-19) in hemodialysis patients: a single-center experience. Exp Clin Transplant. 2020; 18 (3): 275-83.

7. CorbettR.W., BlakeyS., Nitsch D., Loucaidou M., McLeanA., Duncan N., et al. Epidemiology of COVID-19 in an Urban Dialysis Center. J Am Soc Nephrol. 2020; 31 (8): 1815-23.

8. Ma Y., Diao B., Lv X., Zhu J., Liang W., Liu L., et al. 2019 novel coronavirus disease in hemodialysis (HD) patients: report from one HD center in Wuhan China. medRxiv. 2020. DOI: https://doi. org/10.1101/2020.02.24.20027201

9. Li C., Yonglong M., Can T., Dongdong M., Sheng W., Haifeng L., et al. An Analysis on the clinical features of MHD patients with coronavirus disease 2019: a single center study. 2020. DOI: https://doi.org/10.21203/ rs.3.rs-18043/v1

10. Alberici F., Delbarba E., Manenti C., Econimo L., Valerio F., Pola A., et al. Management of patients on dialysis and with kidney transplant during SARS-COV-2 (COVID-19) pandemic in Brescia, Italy. Kidney Int Rep. 2020; 5 (5): 580-5.

11. Bonny V., Maillard A., Mousseaux C., Plaçais L., Richier Q. COVID-19: Pathogenesis of a multi-faceted disease. Rev Med Interne. 2020; 41 (6): 375-89.

12. Interim guidelines "Prevention, diagnosis and treatment of new coronavirus infection (COVID-19)". Version 8.1 (October 1, 2020). Moscow, 2020. (in Russian)

13. Ranieri V.M., Rubenfeld G.D., Thompson B.T., Ferguson N.D. Caldwell E., Fan E., et al. Acute respiratory distress syndrome: the Berlin Definition. JAMA. 2012; 307 (23): 2526-33.

14. Hemmelgarn B.R., Manns B.J., Quan H., Ghali W.A. Adapting the Charlson Comorbidity Index for use in patients with ESRD. Am J Kidney Dis 2003; 42 (1): 125-32.

15. Zhang Y.; The Novel Coronavirus Pneumonia Emergency Response Epidemiology Team. The epidemiological characteristics of an outbreak of 2019 novel coronavirus diseases (COVID-19) - China, 2020. China CDC Weekly. 2020; 2: 113-22

16. Gagliardi I., Patella G., Michael A., Serra R., Provenzano M., Andreucci M. COVID-19 and the kidney: from epidemiology to clinical practice. J Clin Med. 2020; 9 (8): 2506

17. Cheng Y., Luo R., Wang K., Zhang M., Wang Z., Dong L., et al. Kidney disease is associated with in-hospital death of patients with COVID-19. Kidney Int. 2020; 97 (5): 829-38.

18. Wang L., Li X., Chen H., Yan S., Li D., Li Y., et al. Coronavirus dis ease 19 infection does not result in acute kidney injury: an analysis of 116 hospitalized patients from Wuhan, China. Am J Nephrol. 2020; 51 (5) 343-8.

19. Zhang J., Litvinova M., Wang W., Wang Y., Deng X., Chen X., et al. Evolving epidemiology and transmission dynamics of coronavirus disease 2019 outside Hubei province, China: a descriptive and modelling study. Lancet Infect Dis. 2020; 20 (7): 793-802.

20. Boraschi P. COVID-19 Pulmonary involvement: is really an interstitial pneumonia? Acad Radiol. 2020; 27 (6): 900.

21. Henry B.M., Lippi G. Chronic kidney disease is associated with severe coronavirus disease 2019 (COVID-19) infection. Int Urol Nephrol. 2020; 52 (6): 1193-4.

22. Wang R., Liao C., He H., Hu C., Wei Z., Hong Z., et al. COVID-19 in hemodialysis patients: a report of 5 cases. Am J Kidney Dis. 2020; 76 (1): 141-6.

23. Hande A., Ugur M., Ebru H.A.S., Ozlem K.A., Burak S., Sule A., et al. Incidence and immunologic analysis of coronavirus disease (COVID19) in hemodialysis patients: a single-center experience. Exp Clin Transplant. 2020; 18 (3): 275-83.

24. Xiong F., Tang H., Liu L., Tu C., Tian J., Lei C., et al. Clinical characteristics of and medical interventions for COVID-19 in hemodialysis patients in Wuhan, China. J Am Soc Nephrol. 2020; 31 (7): 1387-97.

25. Rombola G., Brunini F. COVID-19 and dialysis: why we should be worried. J Nephrol. 2020; 33 (3): 401-3.

26. Guan W.J., Ni Z.Y., Hu Y., Liang W.H., Ou C.Q., He J.X., et al. Clinical characteristics of coronavirus disease 2019 in China. N Engl J Med. 2020; 392 (18): 1708-20.

27. GrasselliG.,ZangrilloA.,ZanellaA.,AntonelliM.,CabriniL.,CastelliA. et al. COVID-19 Lombardy ICU Network. Baseline characteristics and outcomes of 1591 patients infected with SARS-CoV-2 admitted to ICUs of the Lombardy Region, Italy. JAMA. 2020; 323 (16): 1574-81.

28. Yi Y., Lagniton P.N.P., Ye S., Li E., Xu R.H. COVID-19: what has been learned and to be learned about the novel coronavirus disease. Int J Bio Sci. 2020; 16 (10): 1753-66.

29. Madjid M., Safavi-Naeini P., Solomon S.D., Vardeny O. Potential effects of coronaviruses on the cardiovascular system: a review. JAMA Cardiol. 2020; 5 (7): 831-40. 
30. COVID-19 Registro ERA-EDTA. URL: https://www.era-edta.org/en/ registry/covid-19 (date of access 3 August, 2020)

31. Alberici F., Delbarba E., Manenti C., Econimo L., Valerio F., Pola A., et al. Management of patients on dialysis and with kidney transplantation during the SARS-CoV-2 (COVID-19) pandemic in Brescia, Italy. Kidney Int Rep. 2020; 5 (5): 580-5.

32. Scarpioni R., Manini A., Valsania T., Amicis S.D., Albertazzi V., Melfa L., et al. COVID-19 and its impact on nephropathic patients: the experience at Ospedale "Guglielmo da Saliceto" in Piacenza. G Ital Nefrol. 2020; 37 (2): 1-5.

33. Ma Y., Diao B., Lv X., Zhu J., Liang W., Liu L., et al. 2019 novel coronavirus disease in hemodialysis (HD) patients: report from one HD center in Wuhan China. medRxiv. 2020. DOl: https://doi. org/10.1101/2020.02.24.20027201 (date of access June 17, 2020)

34. Goicoechea M., Luis Camara A.S., Macías N., Morales A.M., Rojas A.G., Bascunana A., et al. COVID-19: clinical course and outcomes of 36 hemodialysis patients in Spain. Kidney Int. 2020; 98 (1): 27-34.
35. Manganaro M., Baldovino S. First considerations on the SARSCoV-2 epidemic in the Dialysis Units of Piedmont and Aosta Valley, Northern Italy. J Nephrol. 2020; 33 (3): 393-5.

36. Singh A.K., Gupta R., Ghosh A., Misra A. Diabetes in COVID-19: prevalence, pathophysiology, prognosis and practical considerations. Diabetes Metab Syndr. 2020; 14 (4): 303-10.

37. Scheen A.J. Obesity and risk of severe COVID-19. Rev Med Suisse. 2020; 16 (695): 1115-9.

38. Li X., Wang L., Yan S., Yang F., Xiang L., Zhu J., et al. Clinical characteristics of 25 death cases with COVID-19: a retrospective review of medical records in a single medical center, Wuhan, China. Int J Infect Dis. 2020; 94: 128-32.

39. Zhou F., Yu T., Du R., Fan G., Liu Y., Liuet Z., et al. Clinical course and risk factors for mortality of adult inpatients with COVID-19 in Wuhan, China: a retrospective cohort study. Lancet 2020; 395 (10 229): 1054-62. 